\title{
Structural diversity and mode of action on lipid membranes of three lactoferrin candidacidal peptides
}

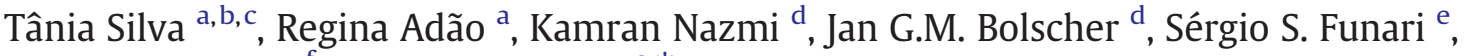 \\ Daniela Uhríková f, Margarida Bastos ${ }^{\mathrm{a}, *}$ \\ a Centro de Investigação em Química CIQ(UP), Department of Chemistry E Biochemistry, Faculty of Sciences, University of Porto, Portugal \\ b IBMC - Instituto de Biologia Molecular e Celular, Universidade do Porto, Portugal \\ c ICBAS - Instituto de Ciências Biomédicas Abel Salazar, Universidade do Porto, Portugal \\ d Academic Centre Dentistry Amsterdam (ACTA), Department of Oral Biochemistry, University of Amsterdam and VU University Amsterdam, Amsterdam, The Netherlands \\ e HASYLAB, DESY, Hamburg, Germany \\ ${ }^{\mathrm{f}}$ Faculty of Pharmacy, Comenius University, Bratislava, Slovak Republic
}

\section{A R T I C L E I N F O}

\section{Article history:}

Received 9 October 2012

Received in revised form 25 January 2013

Accepted 28 January 2013

Available online 4 February 2013

\section{Keywords:}

Antimicrobial peptides

Lactoferrin peptides

DSC

CD

SAXD

\begin{abstract}
A B S T R A C T
The structure and membrane interactions of three antimicrobial peptides from the lactoferrin family were investigated through different techniques. Circular dichroism shows that the peptides adopt a secondary structure in the presence of DMPC/DMPG, and DSC reveals that they all interact with these membranes, albeit differently, whereas only LFchimera has an effect in pure zwitterionic membranes of DMPC. DSC further shows that membrane action is weakest for LFcin17-30, increases for LFampin265-284 and is largest for LFchimera. These differences are clearly reflected in a different structure upon interaction, as revealed by SAX. This technique shows that LFcin17-30 only induces membrane segregation (two lamellar phases are apparent upon cooling from fluid phase), whereas LFampin265-284 induces micellization of the membrane with structure compatible to a micellar cubic phase of space group Pm3n, and LFchimera leads to membrane destruction through the formation of two cubic phases, Pn $3 \mathrm{~m}$ and $\mathrm{Im} 3 \mathrm{~m}$. These structural results show a remarkable parallel with the ones obtained previously by freeze fracture microscopy of the effect of these peptides against Candida albicans.
\end{abstract}

(c) 2013 Elsevier B.V. All rights reserved.

\section{Introduction}

We continue to face a reemergence of infectious diseases, mainly due to the increasing resistance of the pathogens to current therapies and the lack of new and more effective antimicrobial drugs. One potential and interesting alternative to conventional antibiotics is the use of antimicrobial peptides (AMPs). Natural AMPs are present in almost all living organisms as a primary defense mechanism against invading pathogens, with remarkably different structures and bioactivity profiles [1].

\footnotetext{
Abbreviations: AMPs, antimicrobial peptides; CD, circular dichroism; DMPC, dimyristoylphosphatidylcholine; DMPG, dimyristoylphosphatidylglycerol; DSC, differential scanning calorimetry; IMPs, intra-membranous particles; $\mathrm{LC}_{50}$, lethal concentration that causes 50\% of cell death; LFampin265-284, lactoferrampin 265-284; LFchimera, LFcin17-30-K-LFampin265-284; LFcin17-30, lactoferricin 17-30; LUVs, large unilamellar vesicles; MLVs, multilamellar vesicles; P:L, peptide-to-lipid molar ratio; PC, phosphatidylcholine; PE, phosphatidylethanolamine; PG, phosphatidylglycerol; SAXD, small angle $\mathrm{X}$-ray diffraction; WAXD, wide angle X-ray diffraction

* Corresponding author at: Centro Investigação em Química CIQ(UP), Department of Chemistry and Biochemistry, Faculty of Sciences, University of Porto, Rua do Campo Alegre, 4169-007 Porto, Portugal. Tel.: + 351 220402511; fax: + 351220402659.

E-mail address: mbastos@fc.up.pt (M. Bastos).
}

AMPs are considered membrane-active agents leading to cell death by acting on the phospholipid membrane [2]. Within this broad umbrella, it is recognized today that they do not act through a universal mechanism. Different mechanisms have been proposed, consistent with experimental results, providing possible ways for the peptides to disrupt the membrane, leading to cell death. All rely on the same main factor for initial action - adsorption of AMPs onto the membrane due to electrostatic interactions between the cationic peptides and the headgroups of anionic phospholipids. Thereafter, accumulation and positional change eventually lead to the formation of pores, membrane permeabilization or membrane micellization $[1,3-7]$. In some cases internal targets have also been described [5,8-11].

Independently of the details of the mechanism of action the interaction must be as selective as possible regarding the distinction between mammalian cells (higher eukaryotes) and pathogen cells, such as bacteria (prokaryotic cells) or lower eukaryotes as fungi and protozoan. Cytoplasmic membranes of mammalian cells expose predominantly zwitterionic phosphatidylcholine (PC) and sphingomyelin to the extracellular side [1]. On the other hand, cytoplasmic bacterial membranes are mainly composed of zwitterionic phosphatidylethanolamine (PE) and negatively charged phosphatidylglycerol (PG) conferring an overall 
negative charge to the membrane [1,12]. Lower eukaryotes, such as fungi and protozoa also have PC, but they have higher amounts of exposed anionic phospholipids, like phosphatidylserine, than mammalian cells $[1,13,14]$. Indeed it is this differential composition that justifies the unifying electrostatic character of the initial interaction, as well as the ability of the AMPs to act preferentially against pathogens.

Biophysical studies can provide important information on the details of AMPs interaction with the membranes and thus help to unravel their mechanism of action, by providing insight into the effects of the peptides on the membrane structure and information on peptide location. Different techniques have been employed, such as calorimetry, spectroscopy, X-ray diffraction and others [3,12,15-28].

$\mathrm{X}$-ray diffraction studies can give quantitative information on the effects of AMPs on membrane structure, namely if they are capable of altering the phospholipid structure and organization, as well as phase behavior. This information thus allows correlation of these possible changes in lipid polymorphism with models for the mechanism of action of AMPs $[12,22,23,28]$. Growing evidence shows that lipid cubic phases are ubiquitous in the biological world as they have been detected in the plasma membrane of archaebacteria, as well as in the endoplasmic reticulum and mitochondria of mammalian cells. These phases are also involved in biological processes such as membrane fusion, fat digestion and in the reorganization of cell membrane composition [1,29-32]. In the AMP research area, some reports started to appear in the literature indicating the ability of AMPs to induce cubic phases. So far most studies have revealed the existence of bicontinuous (single or double) cubic phases [12,19,24-28,33-35] and a recent one reported a micellar cubic phase [36].

Previously, Bolscher et al. [37,38] have obtained freeze-fracture results on the action of peptides of the lactoferrin family against Candida albicans, showing that the peptides have a quite different effect on the membrane of this pathogen. In the present work we studied the action of these peptides on model membranes of DMPC/DMPG (3:1), considered to be a good model system for C. albicans, by a variety of biophysical techniques. We found that lactoferricin 17-30 (LFcin17-30) induces phase segregation and is the peptide with lowest membrane activity, lactoferrampin 265-284 (LFampin265-284) induces a micellar cubic phase (Pm3n) [36], which to the best of our knowledge is the first experimental evidence of such phase in the context of antimicrobial peptide/membrane interaction. Finally LFchimera, a hybrid peptide between the first two [16], induces two cubic phases of Pn $3 \mathrm{~m}$ and $\mathrm{Im} 3 \mathrm{~m}$ symmetry. These results parallel their effect on C. albicans as derived from freeze-fracture electron microscopy, indicating a remarkable agreement between simple model systems and living organisms.

\section{Materials and methods}

\subsection{Peptide synthesis, purification and characterization}

LFcin17-30, LFampin265-284 and LFchimera were synthesized by solid phase peptide synthesis using Fmoc-protected amino acids (Orpegen Pharma GmbH, Heidelberg, Germany) in a Syro II synthesizer (Biotage, Uppsala Sweden) as described previously [16]. The chimerical peptide comprises a single C-terminal amidated lysine substituted at the $\alpha$ - and $\varepsilon$-amino groups with the two peptides via the C-terminal site and leaving two N-termini as free ends. Peptides were purified to a purity of at least $95 \%$ by semipreparative RP-HPLC (Jasco Corporation Tokyo, Japan) on a Vydac C18-column (218MS510; Vydac, Hesperia, CA, USA) and the authenticity of the peptides was confirmed by MALDI-TOF mass spectrometry on a Microflex LRF mass spectrometer equipped with an additional gridless reflectron (Bruker Daltonik, Bremen, Germany) as described previously [39]. In Table 1 we provide basic information of the peptides, together with their $\mathrm{LC}_{50}$ and their ultrastructural effects against $C$. albicans, as obtained previously by Bolscher et al. [37,38].

\subsection{Preparation of liposomes}

1,2-dimyristoyl-sn-glycero-3-phosphocholine (DMPC) was dissolved in chloroform, and its mixture with 1,2-dimyristoyl-sn-glycero-3[phospho-rac-(1-glycerol)] (DMPG) at a molar ratio of 3:1 was dissolved in chloroform/methanol $(3: 1(\mathrm{v} / \mathrm{v}))$. Both lipids were from Avanti Polar Lipids, Alabama, USA. A film was prepared thereafter in round bottom flasks by drying the sample under a stream of nitrogen, and was kept under vacuum for $3 \mathrm{~h}$ to remove all traces of organic solvents. After drying, the lipid film was first warmed for $30 \mathrm{~min}$ at $\mathrm{ca} .10{ }^{\circ} \mathrm{C}$ above the temperature of the gel-to-liquid crystalline phase transition $\left(T_{\mathrm{m}}\right)$ in a thermostated water bath, and afterwards hydrated with buffer, either HEPES (10 mM HEPES, $100 \mathrm{mM} \mathrm{NaCl}, \mathrm{pH} 7.4$ ) or PBS (9.3 mM, $154 \mathrm{mM} \mathrm{NaCl}, \mathrm{pH}$ 7.2), kept at the same temperature. The multilamellar vesicles (MLVs) were obtained by alternating gentle vortex with short periods in the thermostated water bath at $\sim 35^{\circ} \mathrm{C}$. After this the MLVs were frozen in liquid nitrogen and thawed in a water bath at $35^{\circ} \mathrm{C}$, and this process was repeated 5 times.

Large unilamellar vesicles (LUVs) were obtained from the MLVs by extrusion in a $10 \mathrm{ml}$ stainless steel extruder (Lipex Biomembranes, Vancouver, BC, Canada), inserted in a thermostated cell with a re-circulating water bath, at $35^{\circ} \mathrm{C}$. The samples were passed several times through polycarbonate filters (Nucleopore, Pleasanton, CA, USA) of decreasing pore size $(600,200$ and $100 \mathrm{~nm} ; 5,5$ and 10 times, respectively), under inert $\left(\mathrm{N}_{2}\right)$ atmosphere.

Size distribution of extruded vesicles was determined by qels analysis (Malvern Zeta Sizer 5000, Malvern Instruments, Malvern, Worcestershire,UK) using a helium-neon laser (633 nm) as a source of incident light, and operating at a scattering angle of $90^{\circ}$ and at $37^{\circ} \mathrm{C}$. Mean particle size was thus determined as being of $106 \pm 4 \mathrm{~nm}$ (average and standard deviation of 6 independent measurements). The phospholipid concentration was determined by the phosphomolibdate method [40].

\subsection{Differential scanning calorimetry}

Differential scanning calorimetry (DSC) was performed in a Micro-DSCIII microcalorimeter (SETARAM, Caluire, France) essentially as described previously [41]. In brief, samples were run against HEPES buffer in the reference cell, and blank experiments with HEPES buffer in both cells were also performed for subsequent blank correction. The solution or suspension volume used in each cell was of around $0.8 \mathrm{ml}$, and the masses of solution in sample and reference cells were subsequently matched by weighing $\pm 0.00005 \mathrm{~g}$. Two successive heating and cooling scans were performed for each sample, the heating scan at a scanning rate of $0.5^{\circ} \mathrm{C} / \mathrm{min}$ and the cooling scan at $3{ }^{\circ} \mathrm{C} / \mathrm{min}$, over the temperature range of $10-35{ }^{\circ} \mathrm{C}$. The results provided here always refer to the second heating scan, as we have observed that small differences can exist between first and second scans, but not thereafter. The sample mixtures were prepared immediately before the DSC run, by adding the desired amount of peptide (LFcin17-30, LFampin265-284 or LFchimera) stock solution (in HEPES buffer) to the LUVs suspension of DMPC or DMPC/DMPG (3:1). Samples with peptide-to-lipid molar ratios (P:L) from 1:197 to 1:29 were used. All procedures regarding sample preparation and handling (lag time at low temperature, time between mixtures, and start of the experiment) were kept constant in all experiments, to ensure that all samples had the same thermal history. The instrument was electrically calibrated for temperature and the scan rate with the SETARAM Calibration Unit. The Micro-DSCIII software was used for blank subtraction (run with buffer solution on both cells (sample and reference)). $T_{\mathrm{m}}$ and the $\Delta_{\text {trans }} H$ were calculated by integration of the heat capacity versus temperature curve ( $C p$ versus Temperature). A linear baseline was used to calculate the integral areas under the curves [41]. 
Table 1

Properties of synthetic lactoferrin peptides.

\begin{tabular}{|c|c|c|c|}
\hline Peptide & LFcin17-30 & LFampin265-284 & LFchimera $^{a}$ \\
\hline Sequence & FKCRRWQWRMKKLG & DLIWKLLSKAQEKFGKNKSR & FKCRRWQWRMKKLG-K DLIWKLLSKAQEKFGKNKSR \\
\hline Charge $^{\mathrm{b}}$ & +6 & +4 & +12 \\
\hline Freeze fracture microscopy ${ }^{\mathrm{c}}$ & b & C & d \\
\hline $\mathrm{LC}_{50}^{\mathrm{d}}$ & 1.5 & 1.2 & 0.5 \\
\hline
\end{tabular}

a The carboxyl group of the linking lysine (C-terminal) is in carboxamide form.

b Calculated overall charge at $\mathrm{pH}=7.0$.

c From previously published results [37,38]. Insert (a) is the control. The scale is embedded in each figure.

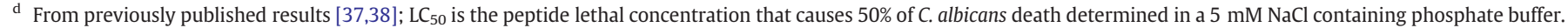

\subsection{Circular dichroism}

Circular dichroism (CD) experiments were carried out in a Jasco 720 spectropolarimeter (Japan Spectroscopy Co., Tokyo) equipped with a rectangular cell, path length of $1 \mathrm{~mm}$. Scans were performed between 175 and $250 \mathrm{~nm}$, bandwidth $1.0 \mathrm{~nm}$, and resolution of 100 mdeg. The measurements were performed in 2 mM HEPES, $100 \mathrm{mM} \mathrm{NaCl}, \mathrm{pH}$ 7.4. Spectra of pure liposome preparations were performed in the same solvent media at the concentrations used in liposome/peptide mixture. These were used as blank experiments to be subtracted from the liposome/peptide spectra. The peptide concentration in buffer was $36 \mu \mathrm{M}$. Liposome concentrations were: $6 \mathrm{mM}$ for DMPC and $3 \mathrm{mM}$ for DMPC/DMPG (3:1), in both cases with a P:L ratio of 1:167. The peptide solution and liposome suspension were mixed just prior to each measurement and incubated at $35{ }^{\circ} \mathrm{C}$ for $30 \mathrm{~min}$ and measurements were performed thereafter at the same temperature. Each spectrum was the average of nine accumulations. After blank correction, the observed ellipticity was converted to a mean residue molar ellipticity $(\theta)\left(\mathrm{deg} \cdot \mathrm{cm}^{2} \cdot \mathrm{dmol}{ }^{-1}\right)$, based on the total amount of peptide in the mixture.

\subsection{X-ray diffraction studies}

Peptide solution in the same buffer used for preparing the liposomes was added to the liposome suspension at different P:L molar ratios, and the mixtures were incubated for $30 \mathrm{~min}$ at $35^{\circ} \mathrm{C}$. The samples were then centrifuged at $13000 \mathrm{rpm}$ in a microcentrifuge at least for $15 \mathrm{~min}$, and transferred thereafter into glass capillaries (Spezialglas Markröhrchen $1.5 \mathrm{~mm}$ capillaries, Glass Technik \& Konstruktion - Müller \& Müller OHG, Germany). In the transfer care was taken to always have a significant amount of supernatant in the capillaries, to guarantee that all samples were studied at high water contents. The capillaries were sealed by flame, and stored at $4{ }^{\circ} \mathrm{C}$, at least 3 days before use.

Small angle X-ray diffraction (SAXD) and wide angle X-ray diffraction (WAXD) experiments were performed at the synchrotron soft condensed matter beamline A2 in HASYLAB at Deutsches Elektronen Synchrotron (DESY), Hamburg, Germany, using a monochromatic radiation of $\lambda=0.15 \mathrm{~nm}$ wavelength. Diffractograms were taken at selected temperatures, where the sample was equilibrated for 5 min before exposure to radiation, or by performing up and down temperature scans at a scan rate $1{ }^{\circ} \mathrm{C} / \mathrm{min}$, where diffractograms were recorded for $10 \mathrm{~s}$ every minute. The heating and cooling of the sample were regulated by a thermocouple connected to the temperature controller JUMO
IMAGO 500 (JUMO GmbH \& Co. KG, Fulda, Germany). The evacuated double-focusing camera was equipped with a linear position sensitive detector for WAXD and a 2D MarCCD detector or a linear position sensitive detector for SAXD. The raw data were normalized against the incident beam intensity. The SAXD patterns were calibrated using Ag behenate [42] or rat tail collagen [43] and the WAXD patterns by tripalmitin or polyethylene terephthalate $[44,45]$. Each diffraction peak was fitted by Lorentzians above a linear background by use of the Peakfit or Origin software programs, in order to derive the lattice parameters. For cubic phases, the lattice parameter was determined as the slope of the dependence of $s\left(\AA^{-1}\right)$ vs. $\sqrt{ }\left(h^{2}+k^{2}+l^{2}\right)$, passing through the origin $(0,0)$, where $h, k, l$ are Miller indices. The uncertainty assigned to the lattice parameters is half of the interval of maximum width that can be obtained through calculation of minimum and maximum values for lattice parameters from the slope above together with its statistical standard error, as obtained from the regression. For lamellar phases, the obtained uncertainty never exceeds $\pm 0.1 \AA$, and thus from hereafter we will not quote the uncertainty for the lattice parameter in lamellar phases. The reported uncertainties for cubic phase lattice parameters are calculated as above and presented together with the lattice parameter value throughout the text.

\section{Results}

\subsection{Differential scanning calorimetry}

DSC experiments show the influence of both lipid charge and peptide-to-lipid molar ratio (P:L) on the thermotropic behavior of the peptide/lipid systems. The endotherms obtained for each peptide, LFcin17-30, LFampin265-284 and LFchimera, with the model membranes of DMPC and DMPC/DMPG (3:1), are shown in Figs. 1 and 2, respectively.

The thermodynamic description is based on the parameters characterizing the gel-to-liquid crystalline phase transition of the liposomes, namely transition temperature $\left(T_{\mathrm{m}}\right)$ and the corresponding enthalpy change $(\Delta H)$. By comparing the calorimetric profiles obtained for the pure liposomes and the liposome/peptide mixtures at the various P:L ratios allow us to derive the influence of the peptides on this thermotropic transition. The obtained thermodynamic parameters are listed in Tables 2 and 3, where values for the pure lipid systems are also shown. In order to overcome the slight differences that may occur for different lipid samples, measurements of the same peptide/lipid system were always performed with liposome suspension from the same preparation batch. 

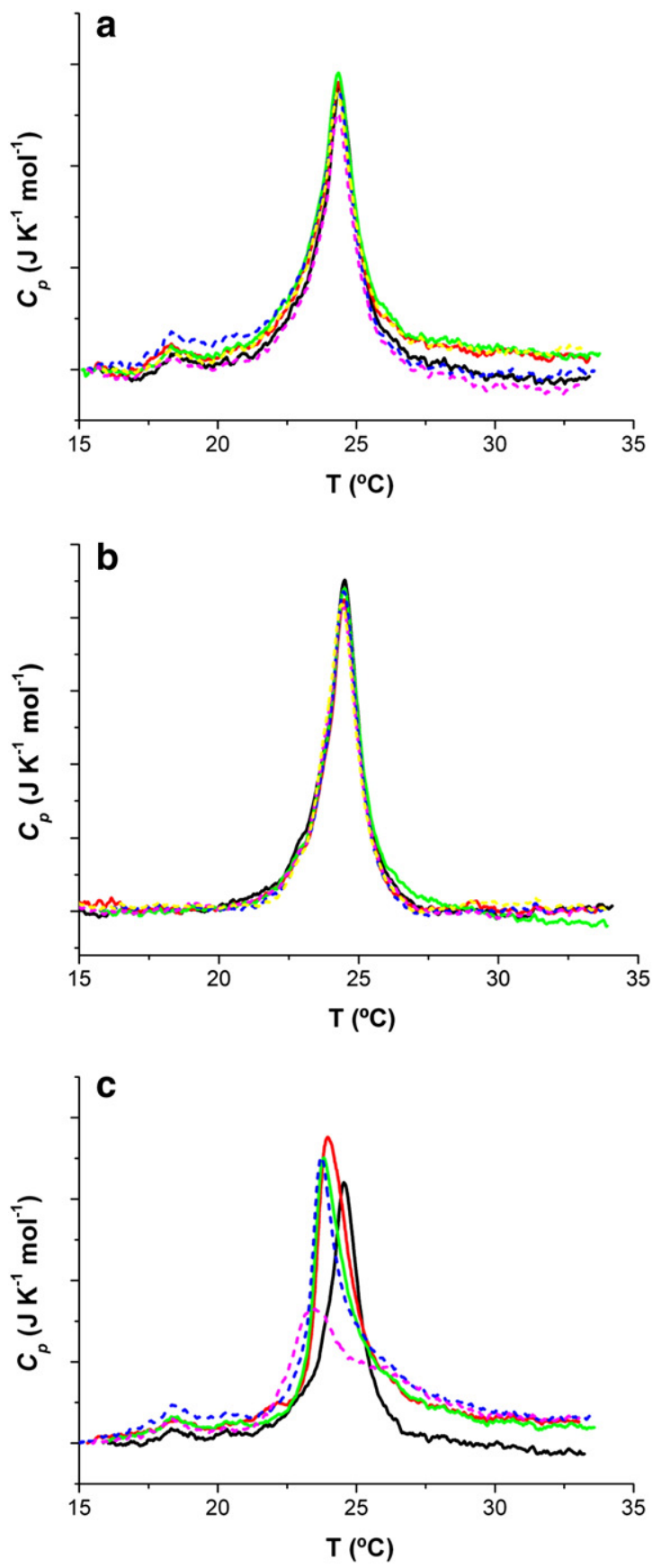

Fig. 1. DSC curves for DMPC and DMPC/peptide mixtures of varying composition. The lipid concentration was 3.0 $\pm 0.3 \mathrm{mM}$ in all experiments. (a) LFcin17-30; (b) LFampin265-284 and (c) LFchimera. Pure lipid (solid black); P:L=1:196 (solid gray); P:L=1:129 (solid light gray); $P: L=1: 96$ (dash black); $P: L=1: 46$ (dash gray); $P: L=1: 29$ (dash light gray). Maximal P:L molar ratio used was 1:46 for LFchimera.

The phase transition observed for LUVs of pure DMPC is characterized by a temperature of $24.5^{\circ} \mathrm{C}$ and an enthalpy change that varies between 19 and $27 \mathrm{~kJ} \cdot \mathrm{mol}^{-1}$ (Table 2 ) for different sample preparations. The $\Delta H_{\text {trans }}$ value observed for the sample used together with LFchimera is significantly higher than the other two (stated uncertainty between samples $\pm 3 \mathrm{~kJ} \cdot \mathrm{mol}^{-1}$ ), but the same sample was used for all mixtures with this peptide, and the results are consistent within sample. The observed thermodynamic parameters are in very good agreement with literature values [41,46,47]. The calorimetric profile for the pure lipid system is a symmetric and cooperative peak. As regarding the peptide/lipid mixtures, we can see that LFcin17-30 and LFampin265-284 do not alter the gel-to-liquid crystalline transition, as
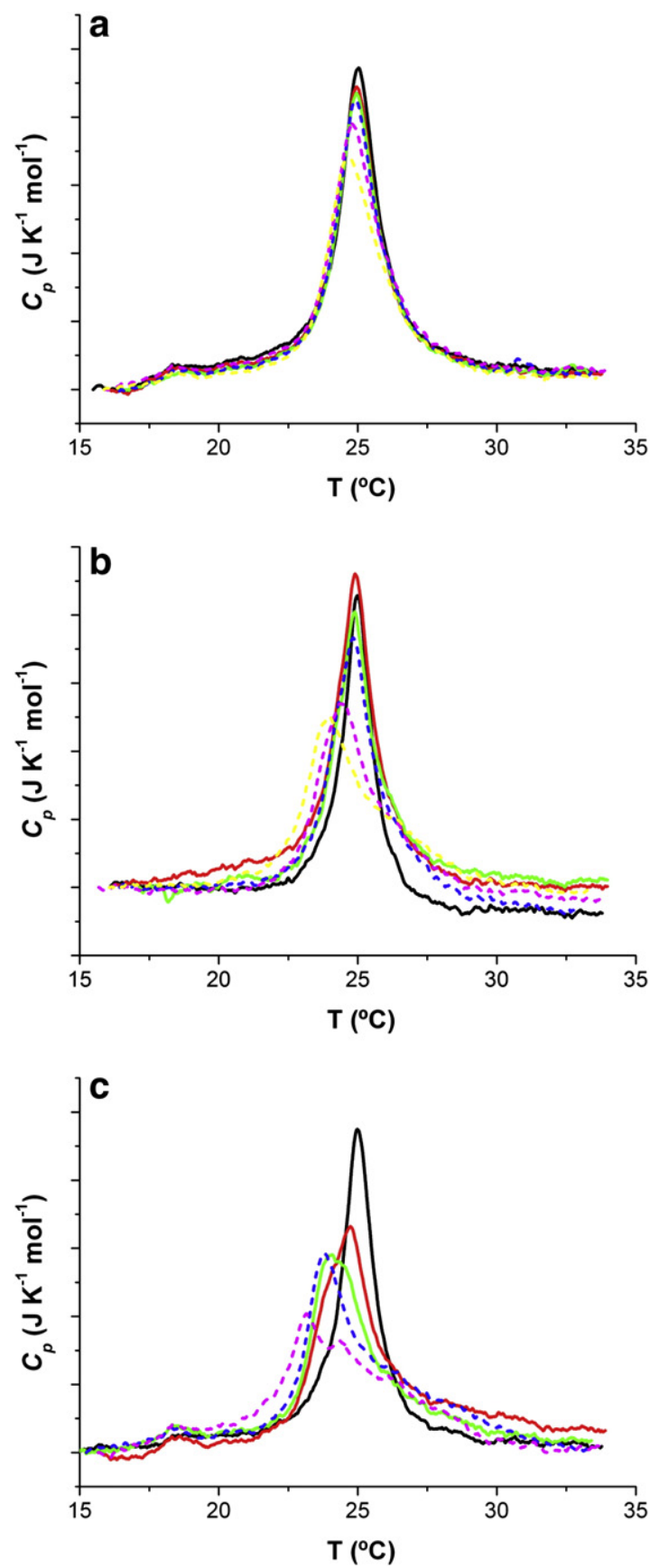

Fig. 2. DSC curves for DMPC/DMPG (3:1) and DMPC/DMPG (3:1)/peptide mixtures of varying composition. The lipid concentration was $3.0 \pm 0.3 \mathrm{mM}$ in all experiments (a) LFcin17-30; (b) LFampin265-284 and (c) LFchimera. Pure lipid (solid black); P:L= 1:196 (solid gray); $\mathrm{P}: \mathrm{L}=1: 129$ (solid light gray); $\mathrm{P}: \mathrm{L}=1: 96$ (dash black); $\mathrm{P}: \mathrm{L}=1: 46$ (dash gray); $\mathrm{P}: \mathrm{L}=1: 29$ (dash light gray). Maximal $\mathrm{P}: \mathrm{L}$ molar ratio used was 1:46 for LFchimera.

the curves are superimposable for all tested P:L ratios and the parameters $T_{\mathrm{m}}$ and $\Delta H_{\text {trans }}$ are the same, within the stated uncertainty (Fig. 1a and $b$, and Table 2). As for LFchimera, it is clear that this peptide interacts with the zwitterionic lipid system for all P:L ratios, and as a result we observe a progressive decrease of $T_{\mathrm{m}}$ as the peptide content in the mixture increases, reflecting a better interaction with the liquid crystalline phase, and the appearance of a shoulder at the high temperature side for the highest P:L ratios (Fig. 1c and Table 2), which is indicative of domain segregation in the membrane [41]. The $\Delta H_{\text {trans }}$ values decrease with increasing peptide content, indicating a progressive 
Table 2

Thermodynamic parameters $T_{\mathrm{m}}$ and $\Delta H_{\text {trans }}$ for the gel to liquid crystalline phase transition of DMPC liposomes for different P:L ratios.

\begin{tabular}{|c|c|c|c|c|c|c|}
\hline \multirow[b]{2}{*}{$(P: L)$} & \multicolumn{3}{|l|}{$T_{\mathrm{m}}{ }^{\mathrm{a}}\left({ }^{\circ} \mathrm{C}\right)$} & \multicolumn{3}{|c|}{$\Delta H_{\text {trans }}{ }^{\mathrm{b}}\left(\mathrm{kJ} \mathrm{mol}^{-1}\right)$} \\
\hline & LFcin17-30 & LFampin265-284 & LFchimera & LFcin17-30 & LFampin265-284 & LFchimera $^{\mathrm{c}}$ \\
\hline 0 & 24.4 & 24.5 & 24.4 & 22 & 19 & 27 \\
\hline $1: 196$ & 24.4 & 24.5 & 23.7 & 21 & 21 & 26 \\
\hline $1: 129$ & 24.4 & 24.5 & 23.6 & 22 & 21 & 23 \\
\hline $1: 96$ & 24.4 & 24.5 & 23.5 & 20 & 19 & 23 \\
\hline $1: 46$ & 24.3 & 24.5 & 23.3 & 20 & 18 & 23 \\
\hline $1: 29$ & 24.3 & 24.4 & - & 21 & 18 & - \\
\hline
\end{tabular}

a $T_{\mathrm{m}}$ estimated uncertainty is $\pm 0.1{ }^{\circ} \mathrm{C}$ (same liposomes preparation, used for the full P:L series) and $\pm 0.3^{\circ} \mathrm{C}$ (within samples).

b $\Delta H_{\text {trans }}$ estimated uncertainty is $\pm 0.5 \mathrm{~kJ} \mathrm{~mol}^{-1}$ (same liposomes preparation, used for the full P:L series) and $\pm 3 \mathrm{~kJ} \mathrm{~mol}^{-1}$ (within samples).

c The value for pure lipid is significantly higher than for the other peptides, but is consistent within the same preparation (series) for this peptide.

destabilization of the membrane organization, and thus probably a deeper insertion of the peptide in the hydrocarbon moiety.

As regarding the mixed system DMPC/DMPG (3:1), we can see that an interaction exists for the three peptides, with strength that increases in the order LFcin17-30, LFampin265-284 and LFchimera (Fig. 2). This is reflected in a very small change in the thermodynamic parameters for LFcin17-30 that is higher for LFampin265-284, and much more significant for LFchimera. In all cases we observe a decrease in $T_{\mathrm{m}}$ and $\Delta H_{\text {trans }}$ (Table 3). For LFcin17-30, although the decrease in both parameters is within the quoted uncertainty, the observed trend is clearly downwards (Fig. 2a). For LFampin265-284 the differences are already outside the error limits at the highest P:L ratios, and overall the trend in both parameters is towards lower values. It should be stressed that for this peptide a significant change in shape occurs already at P:L ratio 1:46, as a very distorted curve with significantly higher width is observed, showing a large reduction in the cooperativity of the transition. This indicates the onset of a significant structural change in the membrane at higher P:L ratios (Fig. 2b).

For LFchimera the decrease in both parameters is significant for all $P: L$ ratios, and although overall the shape of the calorimetric profile is somewhat similar to the one observed for DMPC, it is clear that the effect is much stronger (Fig. 2c). Again we see a trend for $T_{\mathrm{m}}$ and $\Delta H_{\text {trans }}$ to decrease as peptide content in the mixtures increases. This is reasonable, as on one hand the system is still predominantly zwitterionic (DMPC is $75 \%$ of the lipid content of the membrane), and on the other the electrostatic favorable contribution in the case of the partially negatively charged system DMPC/DMPG (3:1) is expected to increase the interaction, as seen in the results. Further, the profile is significantly different from the ones observed for the constituent peptides. We observed the same differential pattern in DSC studies of the three peptides with DMPG liposomes, where LFchimera had a completely disruptive behavior already at low P:L ratios, at odds with the other two peptides, that only presented a significant interaction as the P:L ratio increases [16]. This was also seen by Haney et al. in a DSC study with DPPG liposomes [18]. Again these results are in line with the effect that these peptides show on $C$. albicans, as it will be discussed further.
Finally in order to try to shed some light in the observed lipid/peptide cubic organization for the mixture DMPC/DMPG (3:1) and LFampin265284 at high peptide contents [36] we did perform some further DSC experiments at higher P:L ratios for this system, and the obtained results can be seen in Fig. 3. A clear peak splitting is observed, as anticipated in the highest ratio of previous collection of results. Overall the transitions occur between 20 and $30^{\circ} \mathrm{C}$, and when we did the deconvolution of the overall DSC curves, two peaks were obtained, one at lower and the other at higher temperature as compared to the pure lipid mixture. At 1:12 the first transition is about one degree higher than the ones observed for $1: 8$ and 1:3, whereas the higher temperature transition occurs at about the same temperature (within estimated uncertainty) irrespective of P:L ratio.

\subsection{Circular dichroism}

The secondary structures of LFcin17-30, LFampin265-284 and LFchimera were examined by CD in HEPES buffer and in the presence of the liposome systems. $\mathrm{CD}$ spectra of the peptides at a concentration of $36 \mu \mathrm{M}$ in the aqueous buffer ( $2 \mathrm{mM}$ HEPES, $100 \mathrm{mM} \mathrm{NaCl}, \mathrm{pH}=$ 7.4) are shown in Fig. 4, together with the CD spectra obtained in the presence of DMPC and DMPC/DMPG (3:1) liposomes. Measurements at different peptide concentrations in aqueous buffer showed that the peptide structures are not significantly affected by peptide concentration within the concentration range of 15 to $50 \mu \mathrm{M}$ (results not shown). Therefore, only one concentration is plotted for each peptide in buffer. As for peptide/liposome mixtures, we have tested several combinations, and observed that all provide the same secondary structure information. Therefore, we only plot the ones that provided the best quality spectra.

All peptides show a predominantly random coil structure in buffer. In the presence of DMPC the structure remains practically the same for LFampin265-284, whereas LFcin17-30 changes slightly the minima to higher wavelengths and more negative ellipticity values between 210 and $230 \mathrm{~nm}$, indicating a more significant contribution of $\beta$-structure to the overall CD signal (Fig. $4 a$ and b). In the case of LFchimera, we have a mixture of structures, where the minimum around $200 \mathrm{~nm}$ moves to about $217 \mathrm{~nm}$, characteristic of the presence

Table 3

Thermodynamic parameters $T_{\mathrm{m}}$ and $\Delta H_{\text {trans }}$ for the gel to liquid crystalline phase transition of DMPC/DMPG (3:1) liposomes for different P:L ratios.

\begin{tabular}{|c|c|c|c|c|c|c|}
\hline \multirow[b]{2}{*}{$(P: L)$} & \multicolumn{3}{|l|}{$T_{\mathrm{m}}{ }^{\mathrm{a}}\left({ }^{\circ} \mathrm{C}\right)$} & \multicolumn{3}{|c|}{$\Delta H_{\text {trans }}{ }^{\mathrm{b}}\left(\mathrm{kJ} \mathrm{mol}^{-1}\right)$} \\
\hline & LFcin17-30 & LFampin265-284 & LFchimera & LFcin17-30 & LFampin265-284 & LFchimera \\
\hline 0 & 25.0 & 25.0 & 24.8 & 23 & 21 & 26 \\
\hline $1: 196$ & 25.0 & 24.9 & 24.7 & 22 & 21 & 26 \\
\hline $1: 129$ & 25.0 & 24.9 & 24.5 & 22 & 19 & 24 \\
\hline $1: 96$ & 25.0 & 24.8 & 23.7 & 22 & 19 & 21 \\
\hline $1: 46$ & 24.8 & 24.4 & 23.2 & 22 & 20 & 20 \\
\hline $1: 29$ & 24.6 & 23.9 & - & 21 & 18 & - \\
\hline
\end{tabular}

a $T_{\mathrm{m}}$ estimated uncertainty is $\pm 0.1{ }^{\circ} \mathrm{C}$ (same liposomes preparation, used for the full P:L series) and $\pm 0.3^{\circ} \mathrm{C}$ (within samples).

b $\Delta H_{\text {trans }}$ estimated uncertainty is $\pm 0.5 \mathrm{~kJ} \mathrm{~mol}^{-1}$ (same liposomes preparation, used for the full P:L series) and $\pm 3 \mathrm{~kJ} \mathrm{~mol}^{-1}$ (within samples). 


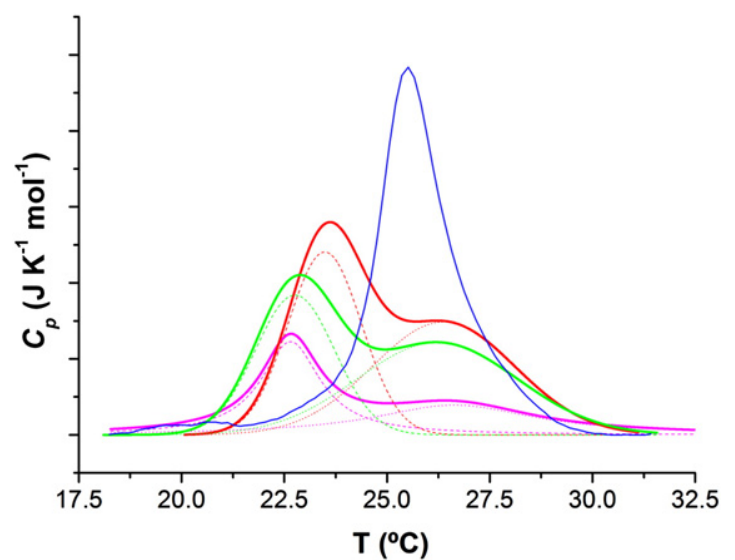

Fig. 3. DSC curves for DMPC/DMPG (3:1) and DMPC/DMPG (3:1)/LFampin 265-284 mixtures at high P:L ratios. The lipid concentration was $6.0 \pm 0.3 \mathrm{mM}$ in all experiments. Pure lipid (solid black); $\mathrm{P}: \mathrm{L}=1: 12$ (heavy solid black); $\mathrm{P}: \mathrm{L}=1: 8$ (heavy solid gray); $\mathrm{P}: \mathrm{L}=1: 3$ (heavy solid light gray). The dashed (low temperature peak) and dotted (high temperature peak) lines represent the deconvoluted curves for each P:L ratio.

of $\beta$-structure (Fig. 4c). This could indicate that although LFcin17-30 and LFampin265-284 alone do hardly interact with DMPC liposomes, as revealed from DSC and CD, when bound together in LFchimera they contribute to the mixture of structures observed by CD for this peptide's interaction with zwitterionic lipids.

In the presence of DMPC/DMPG (3:1), both LFampin265-284 and LFchimera show the predominance of $\alpha$-helix structure, with well-defined minima around 208 and $222 \mathrm{~nm}$ (Fig. 4b and c). Nevertheless the $\alpha$-helix structure is better defined in the case of LFampin265-284. Finally LFcin17-30 shows CD spectra in this lipid mixture that clearly reflects a mixture of $\alpha$-helix and $\beta$-structure (Fig. 4a). We should stress that in a previous paper we showed that this peptide presents the structure of a $\beta$-turn in the presence of purely negatively charged membranes of DMPG [16], and it is thus reasonable that this structure already appears in the DMPC/DMPG (3:1) lipid mixture, although to a smaller extent.

Overall, it is clear that the structure of the peptides changes in the presence of membranes, and that the structure adopted depends on the peptide and the lipid mixture.

\subsection{X-ray diffraction}

Curiously, SAXD experiments revealed that each of the three studied peptides induces different structural changes in DMPC/DMPG (3:1) membrane. The structural changes are temperature dependent, and we present typical diffraction patterns at selected temperatures with the aim to shed more light into the mechanism of peptidemembrane interaction.

The SAXD and WAXD results obtained for LFcin17-30 and DMPC/ DMPG (3:1) at selected temperatures, for a P:L ratio 1:8 $(\mathrm{mol} / \mathrm{mol})$ can be found in Fig. 5. Despite the high peptide to lipid molar ratio in the sample, SAXD did not indicate the occurrence of membrane disruption. As described in the Materials and methods section, prior to interaction with the peptide the DMPC/DMPG (3:1) mixture forms a dispersion of unilamellar (or oligolamellar) vesicles. Such dispersion does not have a long range order and SAX diffraction shows a broad peak with intensity at the level of background (not shown). In the gel phase, the phospholipid acyl chains are fully extended, packed hexagonally and oriented more or less perpendicularly to the surface of the bilayer, a structural feature documented by a peak in WAX diffraction. For our pure DMPC/DMPG (3:1) mixture the temperature of gel to liquid crystalline phase transition was found to be $T_{\mathrm{m}}=25{ }^{\circ} \mathrm{C}$ by our DSC experiments (Table 3). The diffractogram obtained for the mixture LFcin17-30 and DMPC/DMPG (3:1) taken at $10{ }^{\circ} \mathrm{C}$
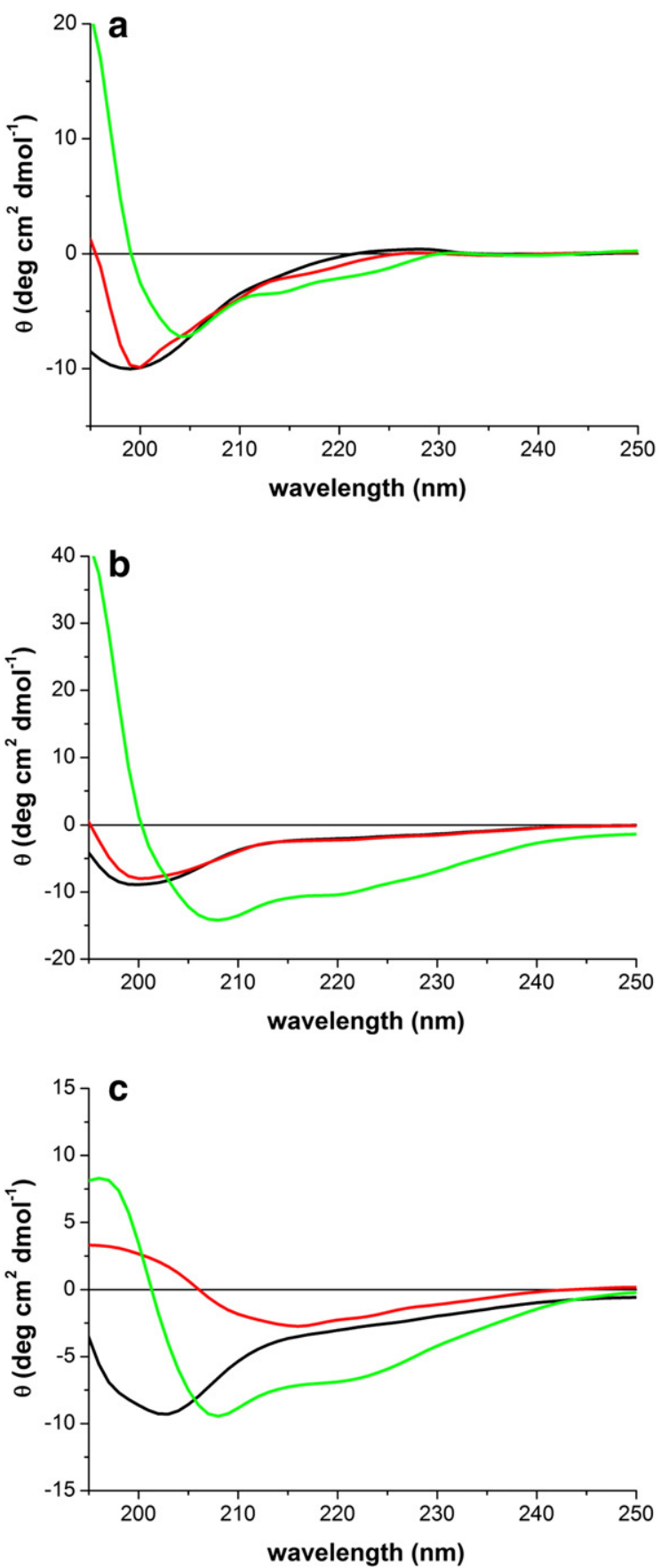

Fig. 4. CD spectra of (a) LFcin17-30, (b) LFampin265-284 and (c) LFchimera at $35{ }^{\circ} \mathrm{C}$ in: buffer (peptide concentration $36 \mu \mathrm{M}$ ) (solid black); DMPC $6 \mathrm{mM}$ (dash gray); and DMPC/DMPG (3:1) $3 \mathrm{mM}$ (dotted light gray). P:L ratio in peptide/LUV mixtures was $1: 167$.

corresponds well with the description above and shows no evidence of interaction of the AMP with the DMPC/DMPG (3:1) membrane. However, heating the system above the phase transition temperature of the mixture, we observe a lamellar phase with a repeat distance $d$ slightly decreasing with increasing temperature $-d=61.7$ and $58.5 \AA$ at 35 and $50{ }^{\circ} \mathrm{C}$, respectively. The WAXD pattern, on the other hand, exhibits wide diffuse scattering in the range $\sim 0.18$ $0.35 \AA^{-1}$, characteristic for phospholipid liquid-like carbon chains (Fig. 5). This indicates that the peptide has a better interaction with the membrane in liquid crystalline resulting in structural changes due to charge screening of both DMPC/DMPG membrane and LFcin17-30. Cooling down the mixture, we identified two distinct 


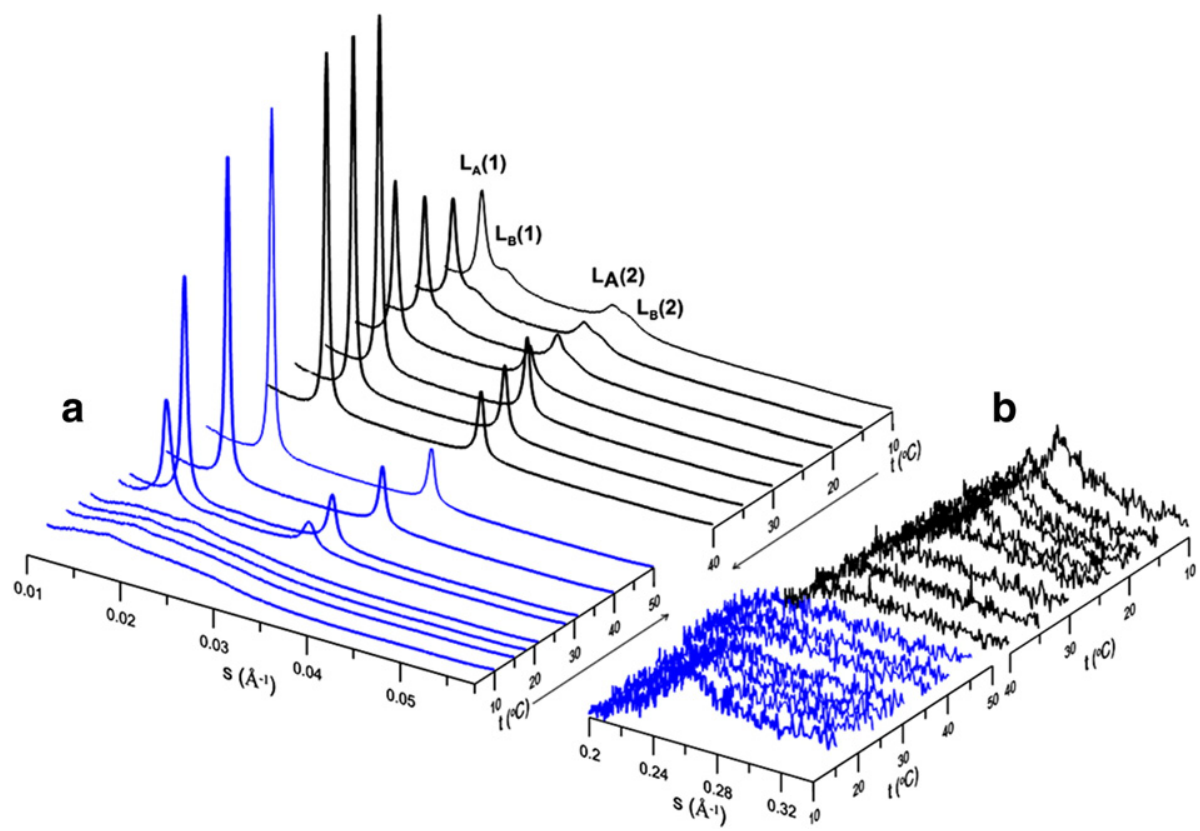

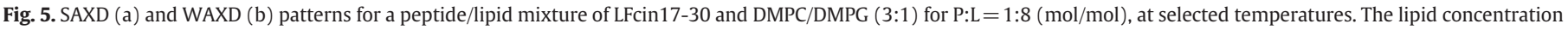
was $12 \mathrm{mM}$.

lamellar phases $\left(\mathrm{L}_{\mathrm{A}}\right.$ and $\left.\mathrm{L}_{\mathrm{B}}\right)$ at temperatures below $T_{m}$ (Fig. 5), with repeat distances $d_{\mathrm{A}}=70.9 \AA$ and $d_{\mathrm{B}}=65.7 \AA$ at $10{ }^{\circ} \mathrm{C}$, an indication that the cooling process leads to a non-homogeneous distribution of the components in the system.

The other two peptides of the lactoferrin family show a more disruptive behavior against the DMPC/DPMG (3:1) membrane. Our previously published X-ray diffraction study on the system with LFampin265-284 and DMPC/DMPG (3:1) revealed the membrane disintegration through a micellar cubic phase of space group Pm3n [36]. By performing SAXD measurements at increasing temperatures we observed that the cubic phase Pm3n occurs in a temperature range where the pure DMPC/DMPG (3:1) mixture was still in the gel state. Fig. 6 shows SAX diffractograms of mixtures of LFampin265-284 with DMPC/DMPG (3:1) at two P:L molar ratios (1:8 and 1:5) and selected temperatures. At both P:L ratios and $\sim 20{ }^{\circ} \mathrm{C}$ we observe a cubic

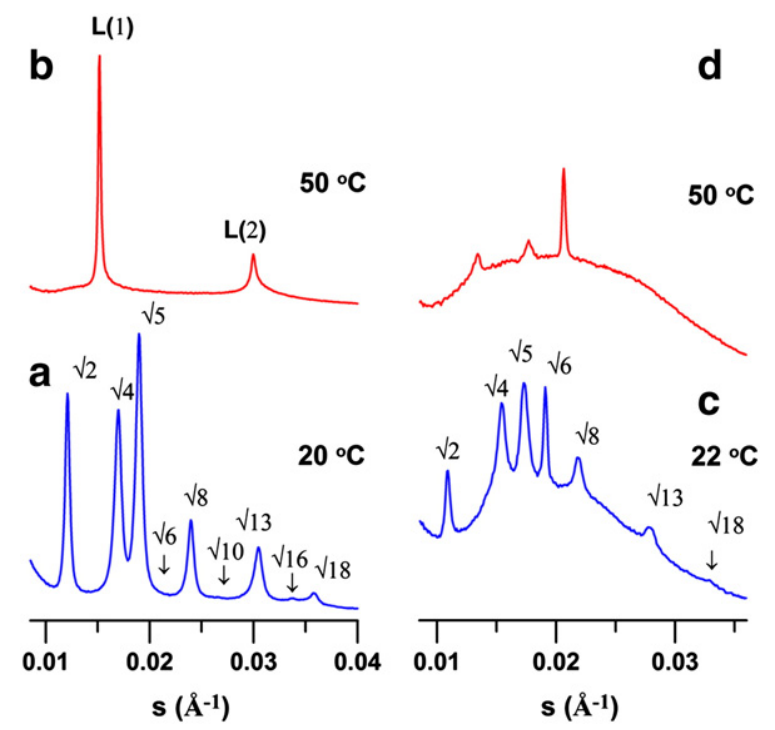

Fig. 6. SAX diffractograms for mixtures of LFampin265-284 with DMPC/DMPG (3:1) at different temperatures and P:L molar ratios: (a) and (b): P:L=1:8; (c) and (d): P:L= 1:5. Intensities are plotted in logarithmic scale. phase of Pm3n space group (Fig. 6a, c). The lattice parameters depend on P:L ratio, as the value obtained for a P:L ratio $1: 5$ was $a=129.0 \pm$ $0.1 \AA$, whereas for $1: 8$ we obtained $a=119.4 \pm 0.1 \AA ̊$, determined as described in the Materials and methods section. The unit cell dimension of Pm3n cubic phase was also found to be dependent on temperature, with the cell dimension slightly decreasing as the temperature increased. The Pm3n phase is usually observed at high water content and is supposed to consist of discrete micelles of amphiphiles arranged on a cubic lattice and separated by a continuous film of water.

The phase transition accompanying the disappearance of the cubic phase involves massive structural changes in the mixture, which in SAX is reflected in a broad, not well resolved peak [36]. At higher temperatures, we detected a lamellar $L_{\alpha}$ phase. The onset of the $L_{\alpha}$ phase was found to depend on $\mathrm{P}: \mathrm{L}$ molar ratio - for $\mathrm{P}: \mathrm{L}=1: 8$ it was observed at $\sim 29^{\circ} \mathrm{C}$, whereas for $\mathrm{P}: \mathrm{L}=1: 5$ the $\mathrm{L}_{\alpha}$ phase was not detected until $\sim 60{ }^{\circ} \mathrm{C}$. Bellow this temperature, diffractograms show 2-3 peaks above a broad background as (Fig. 6), probably due to scattering on partly ordered intermediated structures in the process of cubic phase disappearance.

LFchimera was identified as the peptide with much stronger disruptive effect on the model membrane, as revealed by the DSC results (Section 3.1, Fig. 2c and Table 3).

We examined its effect on the DMPC/DMPG (3:1) membrane at several P:L molar ratios. At low molar ratio $(P: L=1: 38)$ the peptide does not disrupt the membrane, but in the temperature range of $15-40{ }^{\circ} \mathrm{C}$ we observe a lamellar phase with periodicities 64.2 and $56.3 \AA$ at 20 and $40{ }^{\circ} \mathrm{C}$, respectively (not shown). This shows that although the peptide initially interacts with unilamellar or oligolamellar vesicles at low P:L ratio it has the ability to build a multilamellar structure. At the same temperatures, we found $d_{\text {DMPC }}=66.5$ and $62.0 \AA$, where $d_{\text {DMPC }}$ is the repeat distance of DMPC bilayer stacking. The observed lower spacing $(\sim 6 \AA)$ at $40{ }^{\circ} \mathrm{C}$ indicates that either the peptide mediates a closer approach between two opposite charged bilayers due to membrane/AMP charge compensation or the thickness of the membrane itself is reduced after AMP intercalation. As we increase P:L ratio, SAX diffractograms already detected changes in the lamellar organization, but at temperatures below $T_{m}$ and for P:L=1:30 we only observe a not well resolved phase of higher symmetry (not shown), that becomes better defined 


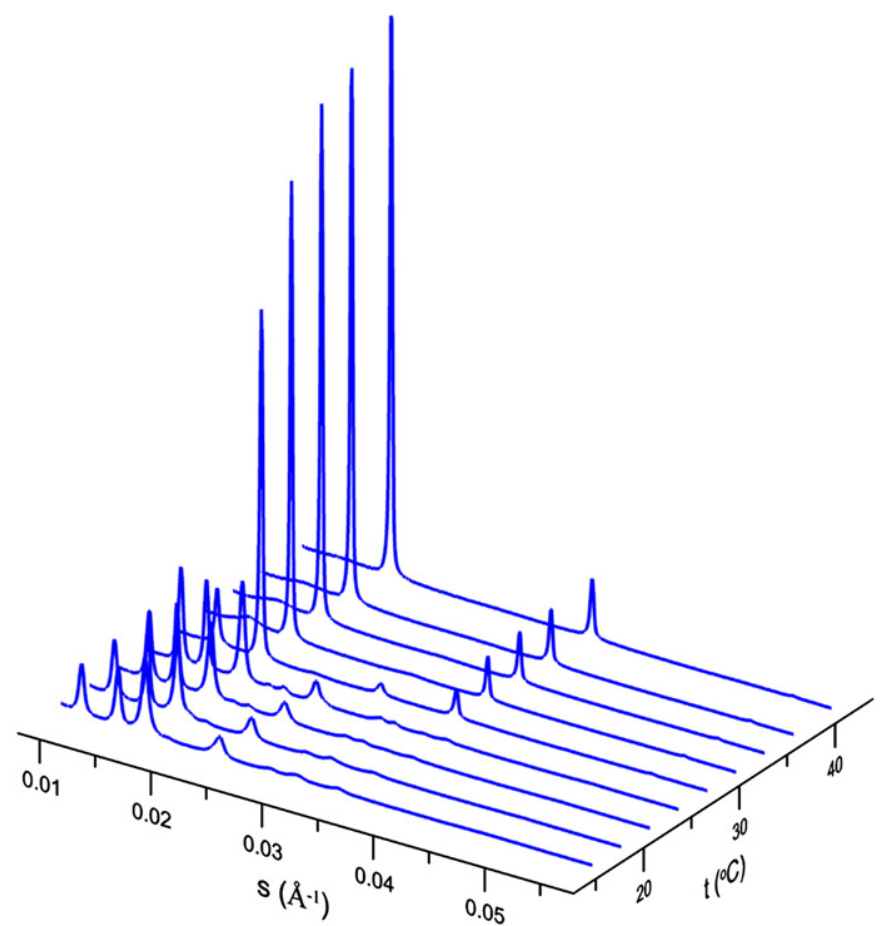

Fig. 7. SAX diffractograms of a heating scan between 15 and $40{ }^{\circ} \mathrm{C}$ for LFchimera and DMPC/DMPG (3:1) at a P:L ratio 1:21.

on cooling. This parallels our previous findings (see above) of a better compatibility with the fluid phase, as expected. Increasing the LFchimera content to molar ratio $P: L=1: 21$, the membrane was disrupted and SAXD clearly confirmed the presence of non-lamellar phase(s) (Figs. 7 and 8). In Fig. 7 we show diffractograms of a heating scan between 15 and $40{ }^{\circ} \mathrm{C}$ for this peptide with DMPC/DMPG (3:1) at this later P:L ratio, where the peaks corresponding to a phase or phases of higher symmetry are clearly seen.

Two representative diffractograms at 24 and $30{ }^{\circ} \mathrm{C}$ with respective peak assignment are presented in Fig. 8. At low temperature, the deconvolution of the diffraction pattern revealed the coexistence of

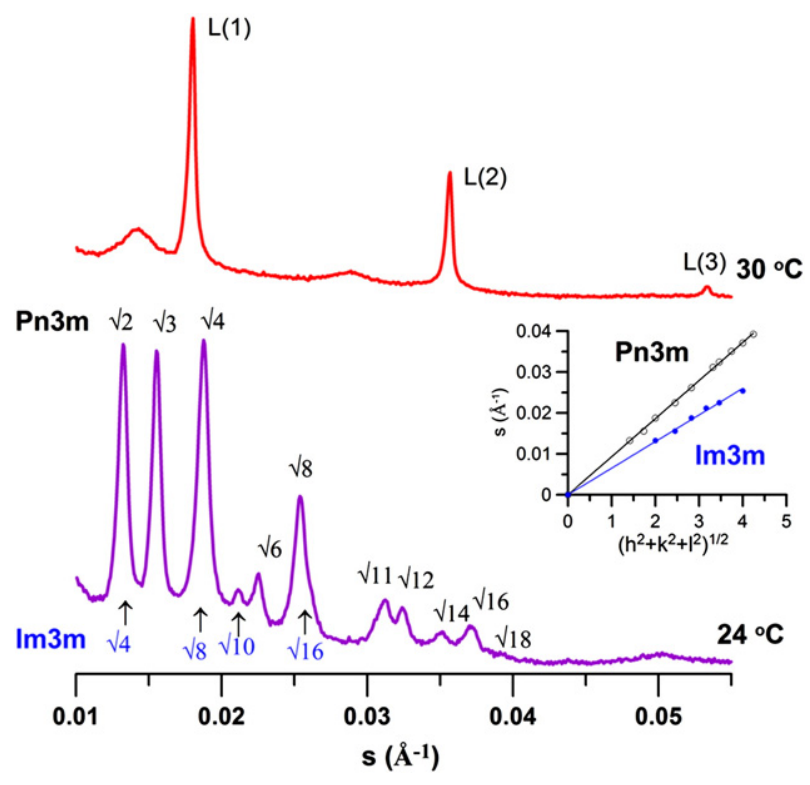

Fig. 8. SAXD patterns for a peptide/lipid mixture of LFchimera and DMPC/DMPG (3:1) for $\mathrm{P}: \mathrm{L}=1: 21(\mathrm{~mol} / \mathrm{mol})$, at selected temperatures, 30 and $24{ }^{\circ} \mathrm{C}$. Insert: a plot of $s\left(\AA^{-1}\right) \mathrm{vs}$. $\sqrt{ }\left(h^{2}+k^{2}+l^{2}\right)$ for all observed reflections at $24{ }^{\circ} \mathrm{C}$. two cubic phases with reflections compatible with Pn3m and $\mathrm{Im} 3 \mathrm{~m}$ space groups. The lattice parameters $a_{\mathrm{Pn} 3 \mathrm{~m}}=107.9 \pm 0.3 \AA$ and $a_{\text {Im3m }}=155.6 \pm 0.9 \AA$ at $24{ }^{\circ} \mathrm{C}$ were determined as described in the Material and methods section, with $\mathrm{R}^{2}=0.9999$ and 0.9997 , respectively. The ratio of the unit cell parameters for the two cubic phases is $a_{\mathrm{Im} 3 \mathrm{~m}} /$ $a_{\text {Pn3m }} \sim 1.4$. On increasing temperature above $\sim 25^{\circ} \mathrm{C}$ both cubic phases vanish and we observe two diffuse peaks with intensities decreasing with temperature, and finally merging in the background (Figs. 7 and 8). Concurrently a lamellar phase is detected, with periodicity $d \sim 56.5-54.9 \AA$ in the range of $27-40{ }^{\circ} \mathrm{C}$, thus decreasing as the temperature increases.

\section{Discussion}

The determination of the structure of AMPs upon interaction with membranes, together with the characterization of the changes observed in the membrane structure as a result of this interaction, can help to unravel the antimicrobial peptide's mechanism of action. In the present study we address these two aspects through DSC, CD and SAXD studies on model membranes, and compare them with previous results of their effects on C. albicans (Table 1) [37,38]. The structural characterization derived from SAXD using model membranes and from freeze-fracture with C. albicans allows a comparison of results derived from two widely different approaches, namely with model and pathogen membranes.

The designed lactoferrin chimera and its constituent peptides presented high candidacidal activity, as can be seen by the low values of $\mathrm{LC}_{50}$ previously obtained $[37,38]$ and provided in Table 1 . Freezefracture electron microscopy of the yeast showed that the peptides induce different levels of membrane damage $[37,38]$. All peptides clearly affected the membrane morphology of C. albicans, albeit differently (Table 1). LFcin17-30 had the weakest effect on the membrane, altering significantly the distribution of the intra-membranous particles (IMP) as compared to control, into IMP-free and IMP-dense areas, suggesting possible IMP aggregation and/or lipid segregation (Table 1, b). LFampin265-284 had a much stronger effect, indicating substantial weakening of the cytoplasmic membrane, as evidenced by the appearance of disruptions into vesicle-like structures (Table 1, c). Finally LFchimera severely destroyed the membrane (Table $1, \mathrm{~d}$ ).

Our DSC results showed that LFcin17-30 and LFampin265-284 do not interact with the model membranes formed from zwitterionic DMPC, whereas LFchimera does. Measurements performed with DPPC liposomes and LFampin265-284 [48] as well as with similar peptides, LFcin17-41 [49], LFcin17-31 [21] and LFampin268-284 [17] also show that the thermotropic profile of the liposomes is not affected by the presence of the peptides, up to a P:L ratio of 1:10. All these results indicate that LFcin and LFampin peptides do not significantly alter the structural organization of bilayers of zwitterionic lipids. It is interesting to note that LFchimera is the result of a special link between LFcin17-30 and LFampin265-284 through an additional lysine [16], and it shows significant interaction with DMPC liposomes, at odds with the constituent peptides. This synergism has been observed for this peptide [16,18,37,50,51], and probably reflects its very high charge $(+12)$, that along with its amphipatic structure leads to the partition to zwitterionic membranes. In line with the critical importance of charge in AMP/membrane interaction, a strong correlation was recently found between the net positive charge of peptides and their capacity to induce anionic lipid clustering, which was found to be independent of their secondary structure [52]. Overall, the obtained results of the interaction of these peptides with zwitterionic membranes are in agreement with the non-hemolytic behavior of LFcin17-30 and LFampin265-284, and the mild hemolytic character of LFchimera [51,53,54].

All three peptides change their secondary structure upon interaction with the membranes, as long as the membrane has significant negative charge ( $25 \%$ in our case), as shown by the CD results. Only in the case of 
LFchimera an altered secondary structure in the presence of pure DMPC membranes is observed, in line with the DSC results. Again this effect might follow from a high charge and amphipaticity, with a mixture of secondary structures that would maximize the electrostatic and hydrophobic interactions.

In the present work we observed the predominance of $\alpha$-helix structure upon interaction with DMPC/DMPG membranes for LFampin265-284 and LFchimera. The presence of significant amounts of $\alpha$-helix was also found for LFampin268-284 [17] and LFampin265$284[18,48]$ in the presence of SDS micelles. Strøm et al. [55] have studied LFcin17-31, and they obtained a percentage of helicity of only $4 \%$ for this peptide in contact with SDS, indicating that in the presence of purely negative micelles this peptide does hardly adopts a helical structure. Haney et al. [18] have studied these three peptides, LFcin17-30, LFampin265-284 and LFchimera by a variety of techniques, but their $\mathrm{CD}$ results are also obtained in the presence of SDS micelles. Again, they found that for LFcin17-30 the intensity of the negative peaks characteristic of $\alpha$-helix structure is fairly weak, whereas for LFampin265284 and LFchimera strong negative minima are present. These results are in line with the ones reported here for DMPC/DMPG (3:1) liposomes, as well as with our previous results in the presence of DMPG [16].

The biological importance of cubic phases was addressed in the seminal work of Luzatti and collaborators [32]. In recent years, Lohner et al. addressed this issue by X-ray scattering in the context of AMPs [12], and the presence of cubic phases upon AMP interaction is now documented both with simple model membranes $[19,36,56]$ as well as with lipid extracts from pathogenic agents [26,27]. Further, their importance has been stressed in recent reviews $[1,23,28]$. The induction of cubic phases by AMPs, mostly by lowering the lamellar to non-lamellar phase boundary of lipid membranes, could indicate that the peptides act by promoting an extensive bilayer curvature, leading eventually to either the formation of pores, mainly of the toroidal type [28] or even to membrane disruption (micellization). The formation of non-lamellar phases could also alter lipid domains in the cellular membrane that are important for many biological processes such endocytosis, or even the activity of membrane proteins that most of the time depend on these domains, culminating into impairment of the membrane function $[33,34]$. Most reported work so far points to the induction of cubic phases, mostly of Pn $3 \mathrm{~m}$ and $\mathrm{Im} 3 \mathrm{~m}$ symmetry, as we have found for LFchimera. In the present report it was very interesting to find that three peptides from the same family, lactoferrin, have quite different effects on membranes of the same composition. LFcin17-30 only induces segregation of the lipid moiety, and leads to the formation of two lamellar phases in the gel phase, on cooling, with repeat distances $d_{\mathrm{A}}=70.9 \AA$ and $d_{\mathrm{B}}=65.7 \AA$ at $10{ }^{\circ} \mathrm{C}$. At this temperature, neutral DMPC itself forms a gel lamellar phase with the repeat distance of $\sim 60.1 \AA$. This value is comparable with the periodicity of $L_{B}$ phase $d_{B}=65.7 \AA$ indicating thus the domain with less AMP. Lowering the temperature beyond $T_{\mathrm{m}}$ may affect differently the mobility of individual membrane's components, which together with the tendency for effective charge compensation results in a segregation of the membrane components. For systems polyelectrolyte-cationic membrane it was suggested that the presence of negatively charged DNA between bilayers can induce a partial lateral segregation of cationic surfactants to minimize the electrostatic energy of the whole system, i.e., lateral "demixing" in the plane of the bilayer can occur [57]. It has been proven experimentally that the polyelectrolytebound population is enriched with oppositely charged lipid, while the polyelectrolyte-free lipid population is correspondingly depleted $[58,59]$. These observations correlate very well with the membrane lipid segregation observed by freeze-fracture, and consequent protein reorganization.

LFcin17-30 was found to be the peptide among the three studied here with lowest membrane activity against $C$. albicans. It is indeed remarkable that our SAX results indicate that for LFcin17-30, only a segregation effect on the lipid mixture takes place, as revealed by the appearance of two lamellar phases upon cooling, and the peptide does not induce a cubic phase. This peptide's mild effect on the membrane also agrees with previous reports of internal targets for LFcin peptides [8-11], as well as with the slower kinetics observed against Leishmania [50]. All these results lead us to propose that peptides that only induce a mild membrane effect, like LFcin17-30, as observed by SAXD (segregation) are likely to have internal targets as part of their mode of action, as long as they are known to be active.

LFampin265-284 induces a micellar cubic phase of Pm3n type on the model membranes of DMPC/DMPG, compatible with a detergent-like action that would cause plasma membrane solubilization [36]. The DSC results obtained for DMPC/DMPG (3:1) and LFampin265-284 at high peptide contents (Fig. 3) allowed us to shed some light in the observed lipid/peptide cubic organization for the mixture. Overall we can see that a transition is still observed by DSC in a temperature range where we already observe the presence of a cubic phase by SAX. As it is known that the transition to cubic phase is a low energy one, thus not easily detectable by DSC [60], the peaks observed must be related to a transition between gel and liquid crystalline phases. This transition is compatible with the peak we observe in WAX at $20{ }^{\circ} \mathrm{C}$ (not shown) indicative of the presence of gel phase. Further, it can account for what we previously hypothesized [36], i.e., that not all lipids are involved in the cubic phase and that the volume fraction of non-lamellar phase depends on temperature and P:L ratio. Thus the results from both methods taken together show that the cubic phase must coexist with a lamellar phase, whose presence is reflected in SAX diffractograms in the high background observed for both 1:5 and 1:8 $\mathrm{P}: \mathrm{L}$ mixtures. Further, by DSC we see that above $30{ }^{\circ} \mathrm{C}$ the mixture is completely in liquid crystalline state, which is compatible with the observed peaks of an organized lamellar phase whose onset we see by SAX at $29{ }^{\circ} \mathrm{C}$ [36] for the $1: 8$ mixture. For the mixture with high peptide content (1:5) the lamellar phase only appears at high temperature, probably because the volume fraction involved in the cubic phase is higher at this peptide content.

As mentioned before in the results' section, two peaks are clearly seen in DSC, thus the mixture is not homogeneous. The first appears at a temperature lower than $T_{\mathrm{m}}$ of the pure lipid mixture, leading to the onset of gel to liquid crystalline transition at $\sim 22.6{ }^{\circ} \mathrm{C}$. This decreased stability should facilitate the transition to a cubic arrangement, and in fact we could see by SAXD that for the mixture 1:8 the onset of cubic phase appearance could be observed already at $\sim 22{ }^{\circ} \mathrm{C}$. The DSC tracing also shows a very broad transition (between $\sim 20$ and $32{ }^{\circ} \mathrm{C}$ ), in a temperature range that coincides with the presence of cubic phase. Thus from $\sim 20^{\circ} \mathrm{C}$ there is a fraction of the peptide/lipid membrane that starts to have a transition to liquid crystalline state and subsequently can undergo a transition to cubic phase in this temperature range, whereas the remaining fraction is still in the gel phase until $\sim 26{ }^{\circ} \mathrm{C}$. This last fraction shows a very broad, non-cooperative transition that could be responsible for the observed high background in the diffractograms in this temperature range. At temperatures above the disappearance of the cubic phase a massive system reorganization takes place, as pointed out before [36]. The observation of $\mathrm{L}_{\alpha}$ at high temperatures can seem surprising, as micellar phase Pm3n is commonly known to change either to a micellar solution or to a hexagonal phase. It must be stressed that we do not suggest that the origin of the $\mathrm{L}_{\alpha}$ phase at high temperature is a transition from the cubic Pm3n phase. As the temperature is raised, the cubic phase vanishes and a redistribution of charged AMP induces massive structural changes. Further, as we suggested earlier [36] and our DSC results here confirm, part of the lipid mixture is not involved in the cubic phase. We found a repeat distance $d=65.9 \AA$ of $\mathrm{L}_{\alpha}$ phase at $50{ }^{\circ} \mathrm{C}(\mathrm{P}: \mathrm{L}=1: 8)$, and DMPC multilamellar vesicles show at this temperature a repeat distance of $60.7 \AA$. The higher spacing observed as compared to pure DMPC can be accounted for either to the presence of the peptide between the lipid bilayers or as due to a residual charge imbalance in the system. 
It is worth noting that the micellization that we could retrieve by SAXD, aside from being the first reported micellar cubic phase in the context of antimicrobial/peptide membrane interactions, is a result that parallels the findings by freeze-fracture for LFampin265-284 when in contact with $C$. albicans, showing again a remarkable agreement between the results with model and actual pathogens' membranes.

SAXD results for LFchimera show the presence of cubic phase, and for the P:L ratio 1:21 the coexistence of two cubic phases (Pn3m and $\mathrm{Im} 3 \mathrm{~m}$ ) was clearly resolved. The ratio obtained of the unit cell parameters for the two cubic phases was $a_{\mathrm{Im} 3 \mathrm{~m}} / a_{\mathrm{Pn} 3 \mathrm{~m}} \sim 1.4$, showing a significant deviation from the ideal Bonnet relation 1.28, for coexisting cubic phases related to the minimal surfaces for a system in thermodynamic equilibrium [61,62]. For AMPs/model membranes, epitaxial relationships between coexisting cubic phases and hexagonal or lamellar phases were reported previously [19,23,26,27,63]. The transitions from liquid crystalline lamellar to cubic phase as well as within different cubic phases occur in a narrow range, and coexistence of phases has been reported, as well as hysteresis. Therefore the system might not have been able to reach thermodynamic equilibrium, and that could justify the deviation of the ratio of lattice parameters from the ideal Bonnet relation. Further, the Bonnet transformation is derived from a mathematical treatment of coexisting infinite minimal surfaces (IMS), and relates the primitive and diamond (and gyroid) surfaces via intermediate self-interacting minimal surfaces, and real systems can deviate from this theoretical prediction, either because they do not conform to it or because the system is not at equilibrium [63]. As the temperature increases above the range of cubic phase stability the lipid network is disrupted. Some released lipid/AMP fragments can initially keep some symmetry in their organization, which in SAXD is observed as two diffuse peaks. With heating, their volume fraction in the sample decreases as documented by the observed decrease in the intensity of the diffuse peaks, while simultaneously we observe the increase in the intensity of peaks related to a lamellar phase. The released lipid or lipid/AMP fragments are reorganized into the lamellar phase to decrease the free energy of the whole system. The retrieved periodicity of the lamellar phase ( $d \sim 54.9 \AA$ at $40{ }^{\circ} \mathrm{C}$ ) is smaller than for DMPC membrane at the same temperature $\left(d_{\mathrm{DMPC}} \sim 62.0 \AA\right)$, and closer to the periodicity $\left(d \sim 56.3 \AA\right.$ at $\left.40{ }^{\circ} \mathrm{C}\right)$ derived from our system at low P:L molar ratio where DMPC/DMPG membrane was not disrupted ( $\mathrm{P}: \mathrm{L}=1: 38)$. At molar ratio $\mathrm{P}: \mathrm{L}=1: 30 \mathrm{SAXD}$ detected the membrane disruption by LFchimera (see Results section). Thus our experiments indicate the value $\sim 1: 34( \pm 4)$ as a critical for the DMPC/DMPG (3:1) membrane disruption by LFchimera.

Finally we would like to point out that the formation of two cubic phases, of symmetry Pn $3 \mathrm{~m}$ and $\mathrm{Im} 3 \mathrm{~m}$, would lead to total membrane disruption, in a way that parallels what was observed by freezefracture. It should be noted that Bolscher et al. [37] refer the presence of some vesicular structures as observed in freeze-fracture experiments also in the case of LFchimera, together with a massive membrane disruption. This peptide is very complex, both due to its extremely high positive charge as well as to its unusual link of constituent peptides through a lysine side chain. In our SAXD experiments with the simple model system of DMPC/DMPG (3:1) we could only assign the presence of two cubic phases, Pn $3 \mathrm{~m}$ and $\mathrm{Im} 3 \mathrm{~m}$. Nevertheless, the possibility of other structures to be formed upon interaction with the more complex membrane of $C$. albicans cannot be discarded.

Thus, overall we could find a remarkable match between the effect of these peptides on $C$. albicans membrane as observed by freezefracture and the structural features they revealed in a simple model membrane system particularly suited to mimic C. albicans (known to have a significant PC content). This has several important consequences: i) it indicates that structural studies involving simple model membranes can provide very important information as regarding different modes of action of AMPs; and ii) the conclusions that can be drawn are indeed much more sound when accompanied by measurements involving real pathogens.

\section{Disclosure statement}

The authors declare that they have no conflict of interest.

\section{Acknowledgements}

This research received funding from the EC's 7th Framework Program (FP7/2007-2013) under grant agreement no. 226716 (HASYLAB project II-20090024 EC), and financial support from FCT/CIQ(UP) to MB and TS, MŠ SR grant VEGA 1/1224/12 to DU, SK-PT-0015-10 to DU, MB and TS, a PhD grant (SFRH/BD/77564/2011) to TS, and a grant from the University of Amsterdam for research into the focal point Oral Infections and Inflammation to JGMB and KN.

\section{References}

[1] V. Teixeira, M.J. Feio, M. Bastos, Role of lipids in the interaction of antimicrobial peptides with membranes, Prog. Lipid Res. 51 (2012) 149-177.

[2] M.A.R.B. Castanho, Membrane-active peptides: methods and results on structure and function, IUL Biotechnology Series, vol. 9, International University Line, La Jolla, CA, USA, 2010, p. 692.

[3] Y. Shai, A. Makovitzky, D. Avrahami, Host defense peptides and lipopeptides: modes of action and potential candidates for the treatment of bacterial and fungal infections, Curr. Protein Pept. Sci. 7 (2006) 479-486.

[4] L.T. Nguyen, E.F. Haney, H.J. Vogel, The expanding scope of antimicrobial peptide structures and their modes of action, Trends Biotechnol. 29 (2011) 464-472.

[5] K.A. Brogden, Antimicrobial peptides: pore formers or metabolic inhibitors in bacteria? Nat. Rev. Microbiol. 3 (2005) 238-250.

[6] H.W. Huang, Action of antimicrobial peptides: two-state model, Biochemistry 39 (2000) 8347-8352.

[7] L. Yang, T.A. Harroun, T.M. Weiss, L. Ding, H.W. Huang, Barrel-stave model or toroidal model? A case study of melittin pores, Biophys. J. 81 (2001) 1475-1485.

[8] H.H. Haukland, H. Ulvatne, K. Sandvik, L.H. Vorland, The antimicrobial peptides lactoferricin B and magainin 2 cross over the bacterial cytoplasmic membrane and reside in the cytoplasm, FEBS Lett. 508 (2001) 389-393.

[9] H. Ulvatne, O. Samuelsen, H.H. Haukland, M. Kramer, L.H. Vorland, Lactoferricin B inhibits bacterial macromolecular synthesis in Escherichia coli and Bacillus subtilis, FEMS Microbiol. Lett. 237 (2004) 377-384.

[10] J.S. Mader, A. Richardson, J. Salsman, D. Top, R. de Antueno, R. Duncan, D.W. Hoskin, Bovine lactoferricin causes apoptosis in Jurkat T-leukemia cells by sequential permeabilization of the cell membrane and targeting of mitochondria, Exp. Cell Res. 313 (2007) 2634-2650.

[11] J.L. Gifford, H.N. Hunter, H.J. Vogel, Lactoferricin: a lactoferrin-derived peptide with antimicrobial, antiviral, antitumor and immunological properties, Cell. Mol. Life Sci. 62 (2005) 2588-2598.

[12] K. Lohner, E.J. Prenner, Differential scanning calorimetry and X-ray diffraction studies of the specificity of the interaction of antimicrobial peptides with membrane-mimetic systems, Biochim. Biophys. Acta 1462 (1999) 141-156.

[13] M.K. Wassef, T.B. Fioretti, D.M. Dwyer, Lipid analyses of isolated surface membranes of Leishmania donovani promastigotes, Lipids 20 (1985) 108-115.

[14] A. Singh, T. Prasad, K. Kapoor, A. Mandal, M. Roth, R. Welti, R. Prasad, Phospholipidome of Candida: each species of Candida has distinctive phospholipid molecular species, OMICS 14 (2010) 665-677.

[15] V.V. Andrushchenko, H.J. Vogel, E.J. Prenner, Interactions of tryptophan-rich cathelicidin antimicrobial peptides with model membranes studied by differential scanning calorimetry, Biochim. Biophys. Acta 1768 (2007) 2447-2458.

[16] J.G. Bolscher, R. Adão, K. Nazmi, P.A. van den Keybus, W. van 't Hof, A.V. Nieuw Amerongen, M. Bastos, E.C. Veerman, Bactericidal activity of LFchimera is stronger and less sensitive to ionic strength than its constituent lactoferricin and lactoferrampin peptides, Biochimie 91 (2009) 123-132.

[17] E.F. Haney, F. Lau, H.J. Vogel, Solution structures and model membrane interactions of lactoferrampin, an antimicrobial peptide derived from bovine lactoferrin, Biochim. Biophys. Acta 1768 (2007) 2355-2364.

[18] E.F. Haney, K. Nazmi, J.G. Bolscher, H.J. Vogel, Structural and biophysical characterization of an antimicrobial peptide chimera comprised of lactoferricin and lactoferrampin, Biochim. Biophys. Acta 1818 (2012) 762-775.

[19] A. Hickel, S. Danner-Pongratz, H. Amenitsch, G. Degovics, M. Rappolt, K. Lohner, G. Pabst, Influence of antimicrobial peptides on the formation of nonlamellar lipid mesophases, Biochim. Biophys. Acta 1778 (2008) 2325-2333.

[20] H.W. Huang, Molecular mechanism of antimicrobial peptides: the origin of cooperativity, Biochim. Biophys. Acta 1758 (2006) 1292-1302.

[21] W. Jing, J.S. Svendsen, H.J. Vogel, Comparison of NMR structures and model-membrane interactions of 15-residue antimicrobial peptides derived from bovine lactoferricin, Biochem. Cell Biol. 84 (2006) 312-326.

[22] C.-C. Lee, Y. Sun, S. Qian, Huey W. Huang, Transmembrane pores formed by human antimicrobial peptide LL-37, Biophys. J. 100 (2011) 1688-1696. 
[23] G. Pabst, D. Zweytick, R. Prassl, K. Lohner, Use of X-ray scattering to aid the design and delivery of membrane-active drugs, Eur. Biophys. J. (2012) 1-15.

[24] E.J. Prenner, R.N. Lewis, R.N. McElhaney, The interaction of the antimicrobial peptide gramicidin $\mathrm{S}$ with lipid bilayer model and biological membranes, Biochim. Biophys. Acta 1462 (1999) 201-221.

[25] E.J. Prenner, R.N. Lewis, K.C. Neuman, S.M. Gruner, L.H. Kondejewski, R.S. Hodges, R.N. McElhaney, Nonlamellar phases induced by the interaction of gramicidin S with lipid bilayers. A possible relationship to membrane-disrupting activity, Biochemistry 36 (1997) 7906-7916.

[26] E. Staudegger, E.J. Prenner, M. Kriechbaum, G. Degovics, R.N. Lewis, R.N. McElhaney, K. Lohner, X-ray studies on the interaction of the antimicrobial peptide gramicidin S with microbial lipid extracts: evidence for cubic phase formation, Biochim. Biophys. Acta 1468 (2000) 213-230.

[27] D. Zweytick, S. Tumer, S.E. Blondelle, K. Lohner, Membrane curvature stress and antibacterial activity of lactoferricin derivatives, Biochem. Biophys. Res. Commun. 369 (2008) 395-400.

[28] E.F. Haney, S. Nathoo, H.J. Vogel, E.J. Prenner, Induction of non-lamellar lipid phases by antimicrobial peptides: a potential link to mode of action, Chem. Phys. Lipids 163 (2010) 82-93.

[29] J. Erbes, C. Czeslik, W. Hahn, R. Winter, M. Rappolt, G. Rapp, On the existence of bicontinuous cubic phases in dioleoylphosphatidylethanolamine, Ber. Bunsenges. Phys. Chem. 98 (1994) 1287-1293.

[30] G. Tresset, The multiple faces of self-assembled lipidic systems, PMC Biophys. 2 (2009) 3.

[31] Z.A. Almsherqi, T. Landh, S.D. Kohlwein, Y. Deng, Chapter 6: cubic membranes the missing dimension of cell membrane organization, Int. Rev. Cell Mol. Biol. 274 (2009) 275-342.

[32] V. Luzzati, Biological significance of lipid polymorphism: the cubic phases, Curr. Opin. Struct. Biol. 7 (1997) 661-668.

[33] K. Lohner, New strategies for novel antibiotics: peptides targeting bacterial cel membranes, Gen. Physiol. Biophys. 28 (2009) 105-116.

[34] K. Lohner, S.E. Blondelle, Molecular mechanisms of membrane perturbation by antimicrobial peptides and the use of biophysical studies in the design of novel peptide antibiotics, Comb. Chem. High Throughput Screen. 8 (2005) 241-256.

[35] S.L. Keller, S.M. Gruner, K. Gawrisch, Small concentrations of alamethicin induce a cubic phase in bulk phosphatidylethanolamine mixtures, Biochim. Biophys. Acta 1278 (1996) 241-246.

[36] M. Bastos, T. Silva, V. Teixeira, K. Nazmi, J.G.M. Bolscher, S.S. Funari, D. Uhríková Lactoferrin-derived antimicrobial peptide induces a micellar cubic phase in a model membrane system, Biophys. J. 101 (2011) L20-L22.

[37] J. Bolscher, K. Nazmi, J. van Marle, W. van 't Hof, E. Veerman, Chimerization of lactoferricin and lactoferrampin peptides strongly potentiates the killing activity against Candida albicans, Biochem. Cell Biol. 90 (2012) 378-388.

[38] M.I. van der Kraan, J. van Marle, K. Nazmi, J. Groenink, W. van 't Hof, E.C. Veerman, J.G. Bolscher, A.V. Nieuw Amerongen, Ultrastructural effects of antimicrobial peptides from bovine lactoferrin on the membranes of Candida albicans and Escherichia coli, Peptides 26 (2005) 1537-1542.

[39] J.G. Bolscher, M.J. Oudhoff, K. Nazmi, J.M. Antos, C.P. Guimaraes, E. Spooner, E.F. Haney, J.J. Garcia Vallejo, H.J. Vogel, W. van't Hof, H.L. Ploegh, E.C. Veerman, Sortase A as a tool for high-yield histatin cyclization, FASEB J. 25 (2011) 2650-2658.

[40] C.W.F. McClare, An accurate and convenient organic phosphorous assay, Anal. Biochem. 39 (1971) 527-530.

[41] F. Abrunhosa, S. Faria, P. Gomes, I. Tomaz, J.C. Pessoa, D. Andreu, M. Bastos, Interaction and lipid-induced conformation of two cecropin-melittin hybrid peptides depend on peptide and membrane composition, J. Phys. Chem. B 109 (2005) 17311-17319.

[42] T.C. Huang, H. Toraya, T.N. Blanton, Y. Wu, X-ray powder diffraction analysis of silver behenate, a possible low-angle diffraction standard, J. Appl. Crystallogr. 26 (1993) 180-184.
[43] N. Roveri, A. Bigi, P.P. Castellani, E. Foresti, M. Marchini, R. Strocchi, Study of rat tail tendon by X-ray diffraction and freeze-etching technics, Boll. Soc. Ital. Biol. Sper. 56 (1980) 953-959.

[44] D. Chapman, The polymorphism of glycerides, Chem. Rev. 62 (1962) 433-456.

[45] M. Kellens, W. Meeussen, H. Reynaers, Crystallization and phase transition studies of tripalmitin, Chem. Phys. Lipids 55 (1990) 163-178.

[46] LIPIDAT, http://www.lipidat.tcd.ie/, (Last access on October 2012).

[47] R.A. Parente, B.R. Lentz, Phase behavior of large unilamellar vesicles composed of synthetic phospholipids, Biochemistry 11 (1984) 2353-2362.

[48] E.F. Haney, K. Nazmi, J.G. Bolscher, H.J. Vogel, Influence of specific amino acid side-chains on the antimicrobial activity and structure of bovine lactoferrampin, Biochem. Cell Biol. 90 (2012) 362-377.

[49] H.J. Vogel, D.J. Schibli, W. Jing, E.M. Lohmeier-Vogel, R.F. Epand, R.M. Epand, Towards a structure-function analysis of bovine lactoferricin and related tryptophan- and arginine-containing peptides, Biochem. Cell Biol. 80 (2002) 49-63.

[50] T. Silva, M.A. Abengozar, M. Fernandez-Reyes, D. Andreu, K. Nazmi, J.G. Bolscher, M. Bastos, L. Rivas, Enhanced leishmanicidal activity of cryptopeptide chimeras from the active N1 domain of bovine lactoferrin, Amino Acids 43 (2012) 2265-2277.

[51] N. Leon-Sicairos, A. Canizalez-Roman, M. de la Garza, M. Reyes-Lopez, J. Zazueta-Beltran, K. Nazmi, B. Gomez-Gil, J.G. Bolscher, Bactericidal effect of lactoferrin and lactoferrin chimera against halophilic Vibrio parahaemolyticus, Biochimie 91 (2009) 133-140.

[52] P. Wadhwani, R.F. Epand, N. Heidenreich, J. Burck, A.S. Ulrich, R.M. Epand, Membrane-active peptides and the clustering of anionic lipids, Biophys. J. 103 (2012) 265-274.

[53] M.I. van der Kraan, J. Groenink, K. Nazmi, E.C. Veerman, J.G. Bolscher, A.V. Nieuw Amerongen, Lactoferrampin: a novel antimicrobial peptide in the N1-domain of bovine lactoferrin, Peptides 25 (2004) 177-183.

[54] M.I. van der Kraan, K. Nazmi, A. Teeken, J. Groenink, W. van 't Hof, E.C. Veerman, J.G. Bolscher, A.V. Nieuw Amerongen, Lactoferrampin, an antimicrobial peptide of bovine lactoferrin, exerts its candidacidal activity by a cluster of positively charged residues at the C-terminus in combination with a helix-facilitating N-terminal part, Biol. Chem. 386 (2005) 137-142.

[55] M.D. Strom, O. Rekdal, W. Stensen, J.S. Svendsen, Increased antibacterial activity of 15 -residue murine lactoferricin derivatives, J. Pept. Res. 57 (2001) 127-139.

[56] K. Lohner, F. Prossnigg, Biological activity and structural aspects of PGLa interaction with membrane mimetic systems, Biochim. Biophys. Acta 1788 (2009) 1656-1666.

[57] R. Bruinsma, J. Mashl, Long-range electrostatic interaction in DNA-cationic lipid complexes, Europhys. Lett. 41 (1998) 165-170.

[58] P. Mitrakos, P.M. MacDonald, DNA induced lateral segregation of cationic amphiphiles in lipid bilayer membranes as detected via 2H NMR, Biochemistry 35 (1996) 16714-16722

[59] D. Uhrikova, M. Hanulova, S.S. Funari, R.S. Khusainova, F. Sersen, P. Balgavy, The structure of DNA-DOPC aggregates formed in presence of calcium and magnesium ions: a small-angle synchrotron X-ray diffraction study, Biochim. Biophys. Acta 1713 (2005) 15-28.

[60] R.N. Lewis, D.A. Mannock, R.N. McElhaney, Differential scanning calorimetry in the study of lipid phase transitions in model and biological membranes: practical considerations, Methods Mol. Biol. 400 (2007) 171-195.

[61] G. Lindblom, L. Rilfors, Cubic phases and isotropic structures formed by membrane lipids - possible biological relevance, Biochim. Biophys. Acta 988 (1989) 221-256.

[62] C. Oguey, J.-F. Sadoc, Crystallographic aspects of the Bonnet transformation for periodic minimal surfaces (and crystals of films), J. Phys. I France 3 (1993) 839-854.

[63] M. Hanulová, Interaction of antimicrobial peptides with lipid membranes, Departments Physik der Universität Hamburg, vol. PhD, Hamburg, Hamburg, 2008. 